\title{
Advance on the nano delivery system of curcumin
}

\author{
Shengjie Tang \\ Leicester International Institute, Dalian University of Technology, Panjin, Liaoning, 124221, China
}

\begin{abstract}
Curcumin has various physiological functions such as anti-oxidation, anti-cancer and prevention of Alzheimer's disease, but its poor water solubility and unstable physicochemical property limits the development and application of curcumin. The nano delivery carriers for curcumin prepared by nanotechnology can effectively solve these problems above. This paper summarized the research reports and literatures at home and abroad in recent years, categorized the nanocarriers of curcumin and analysed the definitions, preparation methods, properties and features, advantages and disadvantages of nano-emulsion, solid lipid particles, liposome, cyclodextrin inclusion compound respectively. In addition, this paper predicted application prospects of curcumin in the fields of food and medicine and provided references for the comprehensive development and utilization of curcumin.
\end{abstract}

\section{Introduction}

Curcumin, a natural polyphenolic compound, is mainly sourced from the roots of Turmeric rhizome. The chemical formula of curcumin is $\mathrm{C}_{21} \mathrm{H}_{20} \mathrm{O}_{6}$, relative molecular weight is 368.39 , melting point is $183^{\circ} \mathrm{C}$, boiling point is $593.2^{\circ} \mathrm{C}$ and its density is $1.307 \mathrm{~g} / \mathrm{cm}^{3}$. It is an orangeyellow crystalline powder which tastes slightly bitter and insoluble in water and ether while soluble in ethanol and propylene glycol, and easily soluble in glacial acetic acid and alkaline solutions whose colour is reddish brown when under alkaline condition, change to yellow when it is under neutral and acidic condition. Each curcumin molecule has two phenolic hydroxyls, which can provide strong resistance to oxidation. Researches showed that the relatively strong antioxidant activity of curcumin can effectively reduce cardiovascular and cerebrovascular diseases and cancer morbidity [1], improve the functionality of brain nerve cells and prevent Alzheimer's Disease [2], meanwhile, protect livers from being affected [3].

Nevertheless, curcumin has very low water solubility [4], and its sensitivity to light, heat and oxygen, and prone to degradation. Only a small amount of curcumin taken orally is absorbed by the intestine and its physiological activity is barely performed [1]. Therefore, the bioavailability of curcumin is very low (about 1\%) [5], which obviously limits its application in the fields of food and medicine.
Under such circumstance, nanotechnology can be used to develop nano delivery carriers for curcumin to eliminate the flaws [6]. Nano delivery carriers can effectively prevent curcumin from being degraded under the conditions of light, heat and oxygen. In addition, the very small diameter of the nanocarriers enables it to carry curcumin through the membrane so that targeting and controlled release are achieved. Besides, because of the relatively high embedding rate and loading capacity, curcumin has better stability and bioavailability. In recent years, nanocomposites, nano-emulsion, nanoliposomes, nanogels, and other delivery carriers made from materials such as starch, oils, proteins, and polysaccharides by using nanotechnology [7]. These nano delivery carriers have not only the superiorities of nano-carrier, but also high biocompatibility, in vivo degradability, security and nontoxicity have important application values and broad market prospects in the development of curcumin delivery carriers.

\section{The nano delivery systems of curcumin}

In the recent years, with the development of nanotechnology, many new types of delivery systems have been designed and made for protecting and deliver curcumin, which are mainly categorized several kinds: nano-emulsion, solid lipid nanoparticles, nanoliposome, micro micelle and cyclodextrin inclusion compound, etc.

\footnotetext{
*Corresponding author Shengjie Tang's e-mail: ST400@student.le.ac.uk
} 


\subsection{Nano-emulsion for loading curcumin}

The diameter of nano-emulsion is usually between 10 and $100 \mathrm{~nm}$. Nano-emulsion is more stable than normal emulsion is because its special rheological properties make phase separation nearly impossible. Researchers compared physical properties of nano-emulsion and ordinary emulsion. The upper layer of ordinary emulsion loaded with curcumin exhibited flavescent turbidity after 4-hour storage at room temperature, while curcuminloaded nano-emulsion is very stable [8]. Borrin et al. [9] used Tween- 80 as the emulsifier and utilized the water, soybean oil and glycerine as basic materials for making nano-emulsion of curcumin which can remain stable after preservation of 60 days. Emulsifier is important for developing nano-emulsion. However, too much emulsifier is not appropriate. To reduce the usage amount of emulsifier (such as Tween-80), some researchers used naturally sourced protein as emulsifier. For example: The curcumin nano-emulsion produced by using whey protein isolate as the emulsifier shows good durability and stability for ionic intensity and heat treatment [10]. Grease is not only an important part of nano-emulsion but is also helpful for the digestion and absorption of curcumin. By comparing digestive rate and how the degree of curcumin in nano-emulsion produced by grease with short, medium and long carbon chains, grease with medium length carbon chain is more suitable for preparing nano-emulsion of curcumin [8].

\subsection{Solid lipid nanoparticle}

Solid lipid nanoparticles are carriers in which solid fat is used as a raw material, hydrophobic drug molecules are embedded inside, and an emulsifier is wrapped outside [11]. The shape of a solid lipid nanoparticle is spherical, and its diameter is usually 50-500 nm [12]. Some available natural materials for preparing solid lipid nanoparticles include triglyceride, glyceryl monostearate, bee wax, animal fat, long carbon chain fatty acid and fatty alcohol, etc. natural emulsifier are usually lecithin, saponin and glucoside. These biologically sourced materials are with relatively excellent biocompatibility, security and degradability.

Curcumin is a hydrophobic drug molecule, and solid lipid nanoparticles can be a good carrier for it. According to previous research reports, a solid lipid nanoparticle with a particle size of about $134.6 \mathrm{~nm}$ was prepared by the emulsification-low temperature solidification method has an embedding rate of $81.92 \%$ for curcumin, and its properties would not change after 12-month storage under $5^{\circ} \mathrm{C}[13]$. In order to prolong the digestion time of solid lipid nanoparticles in the human gastrointestinal tract, scientists tried to use polysaccharides to modify the surface properties of solid lipid nanoparticles to enhance the adsorption capacity of mucosa and improve bioavailability of curcumin when orally taken [14-15]. Ramalingam et al.[16] prepared a kind of solid lipid nanoparticle with a diameter of $451.8 \mathrm{~nm}$, and whose surface is modified by chitosan as the coating, resulting in a larger diameter of $739.26 \mathrm{~nm}$, doubled digestion duration, and the embedding rate and loading amount are also very high. In addition to chitosan, sodium caseinate and pectin can also be used as the surface coating of solid lipid nanoparticles, and the covalent bond is formed between sodium caseinate and pectin, thereby making the physicochemical stability of solid lipid nanoparticles significantly improved, hence curcumin can be digested and absorbed more effectively [17].

\subsection{Liposome}

The liposome is a spherical transmissive carrier of the bimolecular membrane structure, which is self-assembled by molecules, and the liposome has a hydrophilic environment inside, and the bimolecular layer can carry hydrophobic substances. The composition materials of liposome are generally small molecular emulsifier, such as mono-fatty acid glycerides, di-fatty acid glycerides, sodium dodecyl sulfonate, phospholipids, rhamnolipid, saponins, etc. Because liposome has unique bi-molecular membrane structure and physicochemical properties, the prospects for application of liposome are very wide in agriculture, food, medicine and cosmetics [18].

The size of liposome is usually small, the vesicle structure of nanoliposome is typically around 5 to $7 \mathrm{~nm}$. For example: the nanoliposome produced by phospholipid [18], cholesterol and tween 80 as the structural materials has a diameter of $68 \mathrm{~nm}$. When it is used to encapsulate the curcumin, the embedding rate is quite low which is only $57 \%$. Though the embedding rate is a disadvantage, liposome can improve the resistance of curcumin against $\mathrm{pH}$ and metal ions. Besides, this type of nanoliposome can significantly increase the resistance to oxidation and cell permeability of curcumin. To ameliorate the overall properties of liposome, sin the past few years, cholesterol was used to modify the surface of liposome. However, since cholesterol has some bad effects on the blood fat level of human body, researchers started to use some other substances such as polysaccharide to replace cholesterol for preparing liposome [19]. There is a new type of liposome, which is prepared by chitosan and phospholipid and is used for transferring curcumin. The ionic strength resistance of the newly developed nanoliposome is higher than that of nanoliposome only prepared by chitosan, the heat stability is also better than that of nanoliposome prepared solely by phospholipid [20].

As one kind of delivery carrier of curcumin, liposome exhibits some advantages. It can improve the water solubility and cell permeability of the curcumin, prolong the duration in which the curcumin remains in the body and enhance the bioavailability [18,21]. Nevertheless, there are still some drawbacks. Generally, liposome is slightly less stable, the embedding rate is relatively low, and the embedded curcumin is easy to leak, etc. Therefore, many current new researches are dedicating on improving the interface properties of nanoliposome. For instance, chitosan and rhamnolipid are utilized for modifying the nanoliposome to improve its stability and increase the embedding rate and bioavailability [22-23]. 


\subsection{Cyclodextrin inclusion compound}

Cyclodextrin is a cyclic oligosaccharide produced by starch through the action of cyclodextrin glucosyltransferase produced by Bacillus [24]. As shown in the figure $1, \beta$-cyclodextrin crystalline has $7 \mathrm{D}$ galactose, and is enclosed end to end by $\alpha-1,4$ glycosidic bonds. Each glucose is a chair-shaped conformation, and its three-dimensional structure presents a truncated cone- shaped cavity that is hollow at the top and narrow at the top. The hydroxyl of $\beta$-cyclodextrin crystalline all gather at the edge of outside of the molecule, hence the exterior of the molecule has comparatively strong hydrophilia, while the interior of it has hydrophobicity because of the shielding effect of $\mathrm{C}-\mathrm{H}$ bond [25]. Due to the special chemical structure of $\beta$-cyclodextrin crystalline, it can be used as delivery carriers for wrapping curcumin.

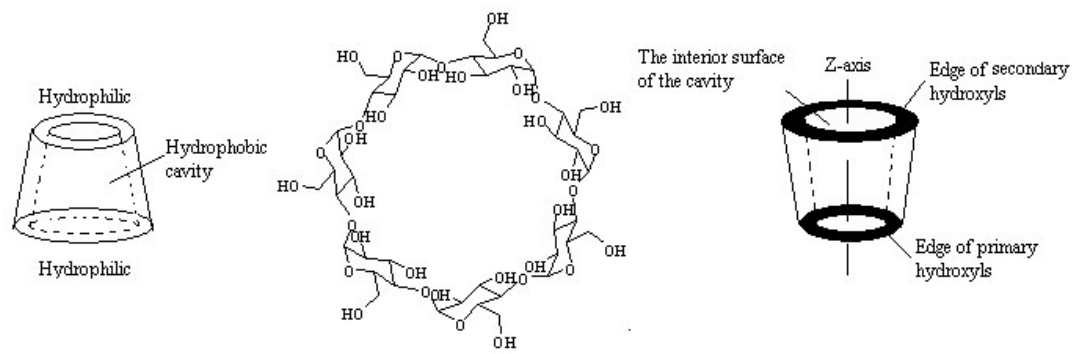

Figure 1. The molecule structure of $\beta$-cyclodextrins.

The process of encapsulating curcumin molecules with cyclodextrin is generally to first prepare a solution of $\beta$-cyclodextrin, then directly add curcumin or an organic solvent solution of curcumin in proportion and mix them thoroughly. For obtaining a better embedding and solubilizing effect, appropriate solvent is also usually chosen to adjust the temperature and $\mathrm{pH}$, ultrasonic assist and other methods are applied [26-27]. Studies have shown that the cyclodextrin inclusion compound can be prepared by applying saturated solution method to achieve the embedding of curcumin, and the cyclodextrin inclusion compound with curcumin can be well dispersed in water [28]. The $\beta$-cyclodextrin derivative obtained by derivatizing $\beta$-cyclodextrin can better embed curcumin and improve its solubility. For example, the delivery carrier prepared by hydroxypropyl- $\beta$-cyclodextrin is superior to $\beta$-cyclodextrin in solubilization effect of curcumin. With the hydroxypropyl $\beta$-cyclodextrin curcumin delivery carriers prepared by the saturated aqueous solution method, the solubility of curcumin in water has increased by 276 times [29], and oral bioavailability has also increased by nearly 3 times [30].

\section{Conclusion}

The solubility of curcumin in water is extremely low. Poor physical and chemical stability and low digestion absorptivity have always been the essential problems that curcumin development is limited. Based on nanotechnology, using proteins, polysaccharides, lipids and cyclodextrins as materials to prepare curcumin nanoemulsion, solid lipid particles, liposomes, cyclodextrin inclusion compounds and other delivery carriers, not only increases curcumin solubility in water, but also protect curcumin from decomposing under light, heat, oxygen and other conditions to achieve the stability of curcumin, and can prolong the duration of curcumin in the human digestive tract to gain a slow release effect so as to improve the bioavailability of curcumin in the human body, thereby expanding the application of curcumin in medicine, food, chemical and other fields.
The nanocarriers of curcumin can not only make better use of physiological activity of curcumin itself, but also able to take advantage of its unique physical and chemical properties to prepare solid and liquid form products. For example, health care milk, functional drinks, solid instant electuary and pills with curcumin nanocarriers can be produced. Based on the efficacy of curcumin on cardiovascular diseases, diabetes and hyperlipoidemia and other chronic diseases, it can be predicted that curcumin nanocarriers have great potential in the development and application of functional foods.

\section{Acknowledgement}

The author wishes to thank the support of my parents and my best friend Qing Yang. They are my motivation for accomplishing this paper and I have been encouraged by them during the writing period.

\section{References}

1. Pulido-Moran M., Moreno-Fernandez J., RamirezTortosa C., et al. (2016) Curcumin and Health. J. Molecules, 21(3): 264-285.

2. Kakkar V., Kaur IP. (2011) Evaluating potential of curcumin loaded solid lipid nanoparticles in aluminium induced behavioural, biochemical and histopathological alterations in mice brain. J. 49(11): 2906-2913.

3. Vera-Ramirez L., Pérez-Lopez P., Varela-Lopez A., et al. (2013) Curcumin and liver disease. J. Biofactors, 39(1): 88-100.

4. Yu H., Ke S., Dong L., et al. (2012) Development of a food-grade organogel with high bioaccessibility and loading of curcuminoids. J. Food Chemistry, 131(1): 48-54.

5. Yang K., Lin L., Tseng T.Y., et al. (2007) Oral bioavailability of curcumin in rat and the herbal 
analysis from Curcuma longa, LC-MS/MS. J. Journal of Chromatography B, 853(1-2): 183-189.

6. Li J., Shin G.H., Lee I.W., et al. (2015) Soluble starch formulated nanocomposite increases water solubility and stability of curcumin. J. Food Hydrocolloids, 56: 41-49.

7. Sergey S., Ramin E.S., Serdar D., et al. (2017) Solubility profiles, hydration and desolvation of curcumin complexed with $\gamma$-cyclodextrin and hydroxypropyl- $\gamma$-cyclodextrin. J. Journal of Molecular Structure, 1134: 91-98.

8. Ahmed K., Li Y., Mcclements D.J., et al. (2012) Nanoemulsion- and emulsion-based delivery systems for curcumin: Encapsulation and release properties. J. Food Chemistry, 132(2): 799-807.

9. Borrin T.R., Georges E. L., Moraes I. C. F., et al. (2016) Curcumin-loaded nanoemulsions produced by the emulsion inversion point (EIP) method: An evaluation of process parameters and physicochemical stability. J. Journal of Food Engineering, 169: 1-9.

10. Li M., Ma Y., Cui J. (2014) Whey-protein-stabilized nanoemulsions as a potential delivery system for water-insoluble curcumin. J. LebensmittelWissenschaft und-Technologie, 59(1): 49-58.

11. Salminen H., Gömmel C., Leuenberger B. H., et al. (2016) Influence of encapsulated functional lipids on crystal structure and chemical stability in solid lipid nanoparticles: Towards bioactive-based design of delivery systems. J. Food Chemistry, 190: 928.

12. Aditya N. P., Ko S. S. (2015) Solid lipid nanoparticles (SLNs): Delivery vehicles for food bioactives. J. Rsc Advances, 5(39): 30902-30911.

13. Kakkar V., Singh S., Singla D., et al. (2011) Exploring solid lipid nanoparticles to enhance the oral bioavailability of curcumin. J. Molecular Nutrition \& Food Research, 55(3): 495.

14. Luo Y., Teng Z., Li Y., et al. (2015) Solid lipid nanoparticles for oral drug delivery: chitosan coating improves stability, controlled delivery, mucoadhesion and cellular uptake. J. Carbohydrate Polymers, 122: 221-229.

15. Ramalingam P., Ko Y. T. (2015) Enhanced Oral Delivery of Curcumin from N -trimethyl Chitosan Surface-Modified Solid Lipid Nanoparticles: Pharmacokinetic and Brain Distribution Evaluations. J. Pharmaceutical research, 32(2): 389.

16. Ramalingam P., Sang W. Y., Ko Y. T. (2016) Nanodelivery systems based on mucoadhesive polymer coated solid lipid nanoparticles to improve the oral intake of food curcumin. J. Food Research International, 84: 113-119.

17. Wang T., Ma X., Lei Y., et al. (2016) Solid lipid nanoparticles coated with cross-linked polymeric double layer for oral delivery of curcumin. J. Colloids \& Surfaces B Biointerfaces, 148:1-11.
18. Chen X., Zou L., Niu J., Liu W., Peng S., Liu C. (2015) The Stability, Sustained Release and Cellular Antioxidant Activity of Curcumin Nanoliposomes. J. Molecules, 20(8): 14293-14311.

19. Karewicz A., Bielska D., Loboda A., et al. (2013) Curcumin-containing liposomes stabilized by thin layers of chitosan derivatives. J. Colloids \& Surfaces B Biointerfaces, 109(9): 307-316.

20. Peng S., Zou L., Liu W., et al. (2017) Hybrid Liposomes Composed of Amphiphilic Chitosan and Phospholipid: Preparation, Stability and Bioavailability as a Carrier for Curcumin. J. Carbohydrate Polymers, 156: 322-332.

21. Takahashi M., Uechi S., Takara K., et al. (2011) Evaluation of an oral carrier system in rats: bioavailability and antioxidant properties of liposome-encapsulated curcumin. J. Journal of Agricultural \& Food Chemistry, 57(19): 9141-6.

22. Catalan-Latorre A., Ravaghi M., Manca M.L., et al. (2016) Freeze-dried eudragit-hyaluronan multicompartment liposomes to improve the intestinal bioavailability of curcumin. J. European Journal of Pharmaceutics \& Biopharmaceutics, 107: 49-55.

23. Moussa Z., Chebl M., Patra D. (2017) Interaction of curcumin with 1,2-dioctadecanoyl-sn-glycero-3phosphocholine liposomes: Intercalation of rhamnolipids enhances membrane fluidity, permeability and stability of drug molecule. J. Colloids \& Surfaces B Biointerfaces, 149: 30-37.

24. Zhong L., Bo C., Hu Y., et al. (2009) Complexation of resveratrol with cyclodextrins: solubility and antioxidant activity. J. Food Chemistry, 113(1): 1720.

25. Karathanos V. T., Mourtzinos I., Yannakopoulou K., et al. (2007) Study of the solubility, antioxidant activity and structure of inclusion complex of vanillin with $\beta$-cyclodextrin. J. Food Chemistry, 101(2): 652658.

26. Chun J., Jo Y. J., Bjrapha P., et al. (2015) Antimicrobial effect of $\alpha$-or $\beta$-cyclodextrin complexes with trans-cinnamaldehyde against Staphylococcus aureus and Escherichia coli. J. Drying Technology, 33(3): 377-383.

27. Su J., Chen J., Li L., et al. (2012) Formation of $\beta$ cyclodextrin inclusion enhances the stability and aqueous solubility of natural borneol. J. Journal of Food Science, 77(6): C658-C664.

28. Haiyee Z. A., Saim N., Said M., et al. (2009) Characterization of cyclodextrin complexes with turmeric oleoresin. J. Food Chemistry, 114(114): 459-465.

29. Jantarat C., Sirathanarun P., Ratanapongsai S., et al. (2014) Curcumin-hydroxypropyl- $\beta$-cyclodextrin inclusion complex preparation methods: effect of common solvent evaporation, freeze drying, and $\mathrm{pH}$ shift on solubility and stability of curcumin. J. 
Tropical Journal of Pharmaceutical Research, 13(8): 1215-1223.

30. Li N., Wang N., Wu T., et al. (2018) Preparation of curcumin-hydroxypropyl- $\beta$-cyclodextrin inclusion complex by cosolvency-lyophilization procedure to enhance oral bioavailability of the drug. J. Drug Development and Industrial Pharmacy, 44(12): 1-24. 